\title{
On Modified Second-order Sliding Mode Synchronization of Two Different Fractional Order Hyperchaotic Systems
}

\author{
Xiaomin Tian, Shumin Fei and Lin Chai \\ Key Laboratory of Measurement and Control of CSE, Ministry of Education, \\ School of Automation, Southeast University, Nanjing 210096, China \\ tianxiaomin100@163.com,smfei@seu.edu.cn,chailin_1@163.com
}

\begin{abstract}
A modified second-order sliding mode (SOSM) control scheme is developed to synchronize two different fractional order hyperchaotic systems. On the basis of chattering free SOSM control scheme, a special fractional sliding mode surface is firstly proposed. Subsequently, a robust control law is designed to ensure the occurrence of sliding mode in a given time. This control scheme is rigorously proved by Lyapunov stability theory. Finally, a numerical example is given to illustrate the effectiveness of the proposed synchronization approach.
\end{abstract}

Keywords: Modified second-order sliding mode control; Fractional order chaotic system; Chaos synchronization; Fractional sliding mode surface

\section{Introduction}

Even though fractional calculus is a mathematical topic with more than 300 years history, its application to physics and engineering has attracted lots of attention only in the recent years. It was found that, with the help of fractional calculus, many systems in interdisciplinary fields can be described delicately, such as viscoelastic system [1], dielectric polarization [2], electrode-electrolyte polarization [3], finance systems and electromagnetic waves [4]. That is to say, fractional derivatives provide a superb instrument for the description of memory and hereditary properties of various materials and processes.

Recently, studying fractional order system has become an active research area. In particular, control and synchronization of the fractional order chaotic systems have attracted much attention from various scientific fields. Some methods have been proposed to achieve chaos synchronization in fractional order chaotic systems. Such as nonlinear feedback control [5], nonlinear state observer control [6], active control [7], adaptive control [8], etc. With the development of sliding mode control (SMC) technique, SMC approach has became a universal method to realize the control or synchronization of fractional order chaotic systems in the past decades [9-11].

However, the traditional sliding mode control is of the first order, and there exists an inevitable drawback when using such traditional SMC, that is the so-called chattering phenomenon, namely, the occurrence of undesirable high frequency vibrations of the system variables which are caused by the discontinuous high frequency nature of firstorder sliding mode control signals. In order to improve the control accuracy and reduce the undesired chattering effect, the second-order sliding mode (SOSM) method is proposed. To the best of our knowledge, few works are available for the synchronization of two different fractional order chaotic systems by using SOSM control approach so far.

In this paper, the synchronization of two different fractional order chaotic systems are researched based on modified SOSM approach. A key point of the introduced approach is the choosing of a special fractional order sliding surface whose first-order total time derivative contains integer order derivatives only, thus being manageable by standard 
sliding mode controllers. The synchronization error system is transformed to a classic control form, which permits a simple stability analysis of sliding mode zero-dynamics.

The rest of this paper is organized as follows. In Section II, relevant definitions and lemmas are presented. Section III gives the main results. In Section IV, a simulation example is used to verify the effectiveness of the proposed method. Finally, conclusions are given in Section V.

\section{Preliminaries}

\subsection{Definitions and Lemmas}

The Riemann-Liouville, Caputo definition are the frequently used definitions of fractional calculus.

Definition 1 The $\alpha$ order Riemann-Liouville fractional integration is given by

$$
{ }_{t_{0}} I_{t}^{\alpha}=\frac{1}{\Gamma(\alpha)} \int_{t_{0}}^{t} \frac{f(\tau)}{(t-\tau)^{1-\alpha}} d \tau
$$

where $\Gamma(\alpha)$ is the Gamma function, determined by

$$
\Gamma(\alpha)=\int_{0}^{\infty} e^{-t} t^{\alpha-1} d t
$$

Definition 2 For $n-1<\alpha \leq n, n \in R$, the $\alpha$ order Riemann-Liouville fractional derivative is defined as

$$
{ }_{t_{0}} D_{t}^{\alpha} f(t)=\frac{d^{\alpha} f(t)}{d t^{\alpha}}=\frac{1}{\Gamma(n-\alpha)} \frac{d^{n}}{d t^{n}} \int_{t_{0}}^{t} \frac{f(\tau)}{(t-\tau)^{\alpha-n+1}} d \tau=\frac{d^{n}}{d t^{n}} I^{n-\alpha} f(t)
$$

Definition 3 The $\alpha$ order Caputo fractional derivative is written as

$$
t_{0} D_{t}^{\alpha} f(t)=\left\{\begin{array}{lc}
\frac{1}{\Gamma(m-\alpha)} \int_{t_{0}}^{t} \frac{f^{(m)}(\tau)}{(t-\tau)^{\alpha-m+1}} d \tau, & m-1<\alpha<m \\
\frac{d^{m}}{d t^{m}} f(t), & \alpha=m
\end{array}\right.
$$

where $m$ is the smallest integer number, larger than $\alpha$.

Lemma 1 (see [12]) Consider the system

$$
\dot{x}(t)=f(x(t)), f(0)=0, x(t) \in R^{n}
$$

where $f: D \rightarrow R^{n}$ is continuous on an open neighborhood $D \subset R^{n}$. Suppose there exists a continuous differential positive definite function $V(x(t)): D \rightarrow R$, real numbers $p>0,0<\eta<1$, such that

$$
\dot{V}(x(t))+p V^{\eta}(x(t)) \leq 0, \forall x(t) \in D
$$


Then the origin of system (5) is a locally finite time stable equilibrium, and the settling time, depending on the initial state $x(0)=x_{0}$, satisfies $T\left(x_{0}\right)=V^{1-\eta}\left(x_{0}\right) / p(1-\eta)$. In addition, if $D=R^{n}$ and $V(x(t))$ is also radially unbounded, then the origin is a globally finite time stable equilibrium of system (5).

Lemma 2 (see [13]) Consider a vector signal $z(t) \in R^{m}$, Let $\alpha \in(0,1)$. If there exist $t_{1}<\infty$ such that $I^{\alpha} z(t)=0, \forall t \geq t_{1}$, then $\lim _{t \rightarrow \infty} z(t)=0$.

Lemma 3 (see [14]) Consider a system $D^{\alpha} x(t)=A x(t)$, the system is asymptotically stable if and only if $|\arg (\operatorname{spec}(A))|>\alpha \pi / 2$, in this case, each state of system converge to zero like $t^{-\alpha}$; The system is stable if and only if either it is asymptotically stable or these eigenvalues which satisfy $|\arg (\operatorname{spec}(A))|=\alpha \pi / 2$ have geometric multiplicity one.

\subsection{Problem Formulation}

Consider the following a class of chaotic systems as drive system

$$
D^{\alpha} x=A_{1} x+g_{1}(x)
$$

where $x \in R^{n}$ is the state vector of the system; $\alpha=\left(\alpha_{1}, \alpha_{2}, \ldots, \alpha_{n}\right)^{T}$ are fractional orders satisfying $0<\alpha_{i} \leq 1 ; A_{1} \in R^{n \times n}$ represents the linear part of the system dynamics; $g_{1}: R^{n} \rightarrow R^{n}$ is the nonlinear part of the system.

The fractional order response systems are described as

$$
D^{\alpha} y=A_{2} y+g_{2}(y)
$$

where $y \in R^{n}$ is the state vector of the system; $\beta=\left(\beta_{1}, \beta_{2}, \ldots, \beta_{n}\right)^{T}$ are satisfying $0<\beta_{i} \leq 1 ; A_{2} \in R^{n \times n}$ and $g_{2}: R^{n} \rightarrow R^{n}$ imply the same roles as $A_{1}$ and $g_{1}$ for the drive system, respectively.

Define $e=y-x$, and add $u(t) \in R^{n}$ to the response system (7), one can get

$$
D^{\beta} y=A_{2} y+g_{2}(y)+u(t)
$$

let

$$
u(t)=u_{1}(t)+u_{2}(t)
$$

where $u_{2}(t) \in R^{n}$ is a vector control function to be designed later, the $u_{1}(t) \in R^{n}$ is a compensation controller, and $u_{1}(t)=D^{\beta} x-A_{2} x-g_{2}(x)$. Substituting (9) into (8), the response system (8) can be rewritten as

$$
D^{\beta} e=A_{2} e+g_{2}(y)-g_{2}(x)+u_{2}(t)
$$

In accordance with the active control design procedure, the nonlinear part of the error dynamics is eliminated by the following choice of the input vector 


$$
u_{2}(t)=g_{2}(x)-g_{2}(y)+B w(t)
$$

Error system (10) is rewritten as

$$
D^{\beta} e=A_{2} e+B w(t)
$$

where $B \in R^{n}$ is a constant gain vector and $w(t)$ is the control input to be designed later by using modified SOSM approach.

Motivated by the above analysis, the synchronization task is equivalently transformed to the asymptotic stabilization problem of the system (12).

We define the sliding manifold for the system (12) in the following form

$$
s=C I^{\beta-1} e
$$

where $C=\left(c_{1}, c_{2}, \ldots, c_{n}\right) \in R^{1 \times n}$ is a constant row vector. From now on the design problem entails the following two steps. In the first step, the sliding manifold is designed. In the second step, the appropriate control input is developed.

\section{Main Results}

In this paper, we research the synchronization problem between incommensurate fractional order hyperchaotic system and commensurate fractional order hyperchaotic system. Considering the special form (13) for the selected sliding manifold, the sliding mode dynamics is actually described by a fractional order system as follows

$$
\begin{aligned}
& D^{\beta} e=A_{2} e+B w(t) \\
& s=C I^{1-\beta} e=0
\end{aligned}
$$

According to the linearity of the fractional integral operator, the sliding manifold can be written in the form

$$
s=I^{1-\beta}(C e)=0
$$

From Lemma 2, when $s$ equal to zero, then $C e$ tend to zero asymptotically.

Next, we will introduce how to design the control input base on modified SOSM approach to stabilize system (14). According to (13), we can get

$$
\dot{s}=C D^{\beta} e
$$

Substituting (14) into (17), it yields

$$
\dot{s}=\mathrm{CA}_{2} e+C B w(t)
$$

We can designed the controller as follows 


$$
\begin{aligned}
& w(t)=(C B)^{-1} v(t) \\
& v(t)=-C A_{2} e-k_{1}|s|^{1 / 2} \operatorname{sgn}(s)-k_{2} s+\rho \\
& \dot{\rho}=-k_{3} \operatorname{sgn}(s)-k_{4} s
\end{aligned}
$$

Considering (18) and (19), it yields

$$
\begin{aligned}
& \dot{s}=-k_{1}|s|^{1 / 2} \operatorname{sgn}(s)-k_{2} s+\rho \\
& \dot{\rho}=-k_{3} \operatorname{sgn}(s)-k_{4} s
\end{aligned}
$$

Next, we will give a theorem to realize the synchronization of two different chaotic systems with nonidentical fractional orders.

Theorem 1 If the appropriate vector $B$ and $C$ are selected such that

$$
\left|\arg \left(\operatorname{spec}\left(\left(I-B(C B)^{-1} C\right) A_{2}\right)\right)\right|>\frac{\beta \pi}{2}
$$

and take the following control law:

$$
\begin{aligned}
& u(t)=u_{1}(t)+u_{2}(t) \\
& u_{1}(t)=D^{\beta} x-A_{2} x-g_{2}(x) \\
& u_{2}(t)=g_{2}(x)-g_{2}(y)+B w(t) \\
& w(t)=(C B)^{-1} v(t) \\
& v(t)=-C A_{2} e-k_{1}|s|^{1 / 2} \operatorname{sgn}(s)-k_{2} s+\rho \\
& \dot{\rho}=-k_{3} \operatorname{sgn}(s)-k_{4} s
\end{aligned}
$$

where $I$ is an identity matrix, $k_{1}, k_{2}, k_{3}, k_{4}>0$ denote the design parameters satisfying that

$$
4 k_{3} k_{4}>\left(k_{4}+9 k_{2}^{2}\right) k_{1}^{2}
$$

then the synchronization error system (14) is globally and asymptotically stable.

Proof Selecting a Lyapunov function for system (20)

$$
V(t)=2 k_{3}|s|+k_{4} s^{2}+\frac{1}{2} \rho^{2}+\frac{1}{2}\left(k_{1}|s|^{1 / 2} \operatorname{sgn}(s)+k_{2} s-\rho\right)^{2}
$$

which can also be written as a quadratic form $V(t)=\xi^{T} P \xi$, where

$$
\begin{aligned}
& \xi=\left(|s|^{1 / 2} \operatorname{sgn}(s) \quad s \quad \rho\right)^{T} \\
& P=\frac{1}{2}\left(\begin{array}{ccc}
4 k_{3}+k_{1}^{2} & k_{1} k_{2} & -k_{1} \\
k_{1} k_{2} & 2 k_{4}+k_{2}^{2} & -k_{2} \\
-k_{1} & -k_{2} & 2
\end{array}\right)
\end{aligned}
$$


It is obviously that $V(t)$ is continuous but is not differentiable at $s=0$; it is positive and radially unbounded if $k_{3}>0$. Besides, we have

$$
\lambda_{\text {min }}(P)\|\xi\|_{2}^{2} \leq V(t) \leq \lambda_{\max }(P)\|\xi\|_{2}^{2}
$$

where $\|\xi\|_{2}^{2}=|s|+s^{2}+\rho^{2}$ is the Euclidean norm of $\xi, \lambda_{\text {min }}(P)$ and $\lambda_{\text {max }}(P)$ are the smallest eigenvalue and the largest eigenvalue of matrix $P$, respectively. Taking the time derivative of $V(t)$ along the system (20), and through a simple derivation, we have

$$
\begin{aligned}
\dot{V}(t)= & -\left(k_{2} k_{3}+2 k_{1}^{2} k_{2}\right)|s|-\left(k_{1} k_{3}+\frac{k_{1}^{3}}{2}\right)|s|^{1 / 2}+k_{1}^{2} \rho \operatorname{sgn}(s) \\
& -\left(k_{1} k_{4}+\frac{5 k_{1} k_{2}^{2}}{2}\right)|s|^{3 / 2}+3 k_{1} k_{2}|s|^{1 / 2} \operatorname{sgn}(s) \rho-\left(k_{2} k_{4}+k_{2}^{3}\right) s^{2} \\
& +2 k_{2}^{2} s \rho-\frac{k_{1}}{2|s|^{1 / 2}} \rho^{2}-k_{2} \rho^{2} \\
= & -\left(k_{2} k_{3}+2 k_{1}^{2} k_{2}\right)|s|+3 k_{1} k_{2}|s|^{1 / 2} \operatorname{sgn}(s) \rho-\left(k_{2} k_{4}+k_{2}^{3}\right) s^{2}+2 k_{2}^{2} s \rho-k_{2} \rho^{2} \\
& -\frac{1}{|s|^{1 / 2}}\left[\left(k_{1} k_{3}+\frac{k_{1}^{3}}{2}\right)|s|-k_{1}^{2}|s|^{1 / 2} \operatorname{sgn}(s) \rho+\left(k_{1} k_{4}+\frac{5 k_{1} k_{2}^{2}}{2}\right) s^{2}+\frac{k_{1}}{2} \rho^{2}\right]
\end{aligned}
$$

Therefore, we can rewrite $\dot{V}(t)$ as

$$
\dot{V}(t)=-\frac{1}{|s|^{1 / 2}} \xi^{T} \Omega_{1} \xi-\xi^{T} \Omega_{2} \xi
$$

Where

$$
\begin{aligned}
& \Omega_{1}=\left(\begin{array}{ccc}
k_{1} k_{3}+\frac{1}{2} k_{1}^{3} & 0 & -\frac{1}{2} k_{1}^{2} \\
0 & k_{1} k_{4}+\frac{5}{2} k_{1} k_{2}^{2} & 0 \\
-\frac{1}{2} k_{1}^{2} & 0 & \frac{1}{2} k_{1}
\end{array}\right), \\
& \Omega_{2}=\left(\begin{array}{ccc}
k_{2} k_{3}+2 k_{1}^{2} k_{2} & 0 & -\frac{3}{2} k_{1} k_{2} \\
0 & k_{2} k_{4}+k_{2}^{3} & -k_{2}^{2} \\
-\frac{3}{2} k_{1} k_{2} & -k_{2}^{2} & k_{2}
\end{array}\right)
\end{aligned}
$$

Note that $\dot{V}(t)$ is negative definite if $\Omega_{1}>0$ and $\Omega_{2}>0$. It is not difficult to show that this will be the case if $k_{i}>0, i=1,2,3,4$ and

$$
4 k_{3} k_{4}>\left(k_{4}+9 k_{2}^{2}\right) k_{1}^{2}
$$

then we have $\dot{V}(t)<0$. 
Since

$$
\begin{gathered}
\dot{V}(t) \leq-\frac{1}{|s|^{1 / 2}} \lambda_{\text {min }}\left(\Omega_{1}\right)\|\xi\|_{2}^{2}-\lambda_{\text {min }}\left(\Omega_{2}\right)\|\xi\|_{2}^{2} \\
\|\xi\|_{2}^{2}=|s|+s^{2}+\rho \\
\lambda_{\text {min }}(P)\|\xi\|_{2}^{2} \leq V(t) \leq \lambda_{\text {max }}(P)\|\xi\|_{2}^{2}
\end{gathered}
$$

So, one can get

$$
\begin{gathered}
|s|^{1 / 2} \leq\|\xi\|_{2} \leq \frac{\sqrt{V(t)}}{\sqrt{\lambda_{\min }(P)}} \\
\frac{V(t)}{\lambda_{\max }(P)} \leq\|\xi\|_{2}^{2} \leq \frac{V(t)}{\lambda_{\min }(P)}
\end{gathered}
$$

It follows that

$$
\begin{aligned}
\dot{V}(t) & \leq-\lambda_{\text {min }}\left(\Omega_{1}\right) \cdot \frac{\sqrt{\lambda_{\text {min }}(P)}}{\sqrt{V(t)}} \cdot \frac{V(t)}{\lambda_{\text {max }}(P)}-\lambda_{\text {min }}\left(\Omega_{2}\right) \frac{V(t)}{\lambda_{\text {max }}(P)} \\
& =-\gamma_{1} V^{1 / 2}(t)-\gamma_{2} V(t) \leq-\gamma_{1} V^{1 / 2}(t)
\end{aligned}
$$

By Lemma 1, it follows easily that the $V(t)$ Lyapunov function and $s$ globally converge to zero in finite time. When the sliding mode variable $s$ equal to zero, according to the third equation of (19) we can find a constant $M$ such that $\rho=M$, substituting (19) into (14) we can get

$$
D^{\alpha} e=\left(I-B(C B)^{-1} C\right) A_{2} e+B(C B)^{-1} M
$$

According to Ref. [15], we can regard (34) as a linear system with bounded input $B(C B)^{-1} M$. Furthermore, according to Lemma 3, system (34) is asymptotically stable if and only if $\left|\arg \left(\operatorname{spec}\left(\left(I-B(C B)^{-1} C\right) A_{2}\right)\right)\right|>\beta \pi / 2$, that is the synchronization of two different hyperchaotic systems with different fractional orders is achieved. Hence, the proof is completed.

\section{Numerical Simulation}

According to the numerical algorithm of fractional order systems presented in [16], we can research the synchronization problem between fractional order hyperchaotic Lorenz system and a novel hyperchaotic system [17].

First, we take the fractional order hyperchaotic Lorenz system as drive system, which is written as

$$
\begin{aligned}
& D^{\alpha_{1}} x_{1}=a\left(x_{2}-x_{1}\right)+x_{4} \\
& D^{\alpha_{2}} x_{2}=c x_{1}-x_{2}-x_{1} x_{3} \\
& D^{\alpha_{3}} x_{3}=x_{1} x_{2}-b x_{3} \\
& D^{\alpha_{4}} x_{4}=-x_{2} x_{3}-r x_{4}
\end{aligned}
$$

On the basis of Ref. [16], the drive system can be discrete in the following form 


$$
\begin{aligned}
& x_{1}(n+1)=x_{1}(0)+\frac{h^{\alpha_{1}}}{\Gamma\left(\alpha_{1}+2\right)}\left[a\left(x_{2}^{p}(n+1) x_{1}^{p}(n+1)\right)+x_{4}^{p}(n+1)+\sum_{j=0}^{n} \xi_{j, n+1}\left(a\left(x_{2}(j) x_{1}(j)\right)+x_{4}(j)\right)\right] \\
& x_{2}(n+1)=x_{2}(0)+\frac{h^{\alpha_{2}}}{\Gamma\left(\alpha_{2}+2\right)}\left[c x_{1}^{p}(n+1)-x_{2}^{p}(n+1)-x_{1}^{p}(n+1) x_{3}^{p}(n+1)+\sum_{j=0}^{n} \xi_{j, n+1}\left(c x_{1}^{p}(j)-x_{2}^{p}(j)-x_{1}^{p}(j) x_{3}^{p}(j)\right)\right] \\
& x_{3}(n+1)=x_{3}(0)+\frac{h^{\alpha_{3}}}{\Gamma\left(\alpha_{3}+2\right)}\left[x_{1}^{p}(n+1) x_{2}^{p}(n+1)-b x_{3}^{p}(n+1)+\sum_{j=0}^{n} \xi_{j, n+1}\left(x_{1}^{p}(j) x_{2}^{p}(j)-b x_{3}^{p}(j)\right)\right] \\
& x_{4}(n+1)=x_{4}(0)+\frac{h^{\alpha_{4}}}{\Gamma\left(\alpha_{4}+2\right)}\left[-x_{2}^{p}(n+1) x_{3}^{p}(n+1)-r x_{4}^{p}(n+1)+\sum_{j=0}^{n} \xi_{j, n+1}\left(-x_{2}^{p}(j) x_{3}^{p}(j)-r x_{4}^{p}(j)\right)\right]
\end{aligned}
$$

in which $x_{l}^{p}(n+1)$ is the predictor of $x_{l}(n+1)$, determined by

$$
\begin{aligned}
& x_{1}^{p}(n+1)=x_{1}(0)+\frac{1}{\Gamma(\alpha)} \sum_{j=0}^{n} \eta_{j, n+1}\left[a\left(x_{2}(j) x_{1}(j)\right)+x_{4}(j)\right] \\
& x_{2}^{p}(n+1)=x_{2}(0)+\frac{1}{\Gamma(\alpha)} \sum_{j=0}^{n} \eta_{j, n+1}\left[c x_{1}(j)-x_{2}(j)-x_{1}(j) x_{3}(j)\right] \\
& x_{3}^{p}(n+1)=x_{3}(0)+\frac{1}{\Gamma(\alpha)} \sum_{j=0}^{n} \eta_{j, n+1}\left[x_{1}(j) x_{2}(j)-b x_{3}(j)\right] \\
& x_{4}^{p}(n+1)=x_{4}(0)+\frac{1}{\Gamma(\alpha)} \sum_{j=0}^{n} \eta_{j, n+1}\left[-x_{2}(j) x_{3}(j)-r x_{4}(j)\right]
\end{aligned}
$$

where

$$
\begin{gathered}
\xi_{j, n+1}= \begin{cases}n^{\alpha+1}-(n-\alpha)(n+1)^{\alpha}, & j=0 \\
(n-j+2)^{\alpha+1}+(n-j)^{\alpha+1}-2(n-j+1)^{\alpha+1}, 1 \leq j \leq n \\
1, & j=n+1\end{cases} \\
\eta_{j, n+1}=\frac{h^{\alpha}}{\alpha}\left((n+1-j)^{\alpha}-(n-j)^{\alpha}\right), \quad 0 \leq j \leq n
\end{gathered}
$$

when $a=10, b=8 / 3, c=28, r=1, \alpha=(0.99,0.98,0.97,0.98)^{T}$, with the initial condition $x(0)=(1,-2,3,1)^{T}$, the system (35) is hyperchaotic. The chaotic attractors are shown in Figure 1 (red line).

We take a novel hyperchaotic system [17] as response system, which is described by

$$
\begin{aligned}
& D^{\beta_{1}} y_{1}=a_{1}\left(y_{2}-y_{1}\right) \\
& D^{\beta_{2}} y_{2}=q y_{1}+c_{1} y_{4}+y_{1} y_{3} \\
& D^{\beta_{3}} y_{3}=-b_{1} y_{3}-d_{1} y_{4}-2 y_{1}^{2}-2 y_{2}^{2} \\
& D^{\beta_{4}} y_{4}=-r_{1} y_{1}
\end{aligned}
$$

Similarly, through the same operation, we can conclude that when $a_{1}=10, b_{1}=2.5$, $c_{1}=1, r_{1}=10, q=40, \beta=(0.98,0.98,0.98,0.98)^{T}$, with the initial condition $y(0)=(-1,-3,6,1)^{T}$, the attractors of response system are shown in Figure 1 (blue line). 

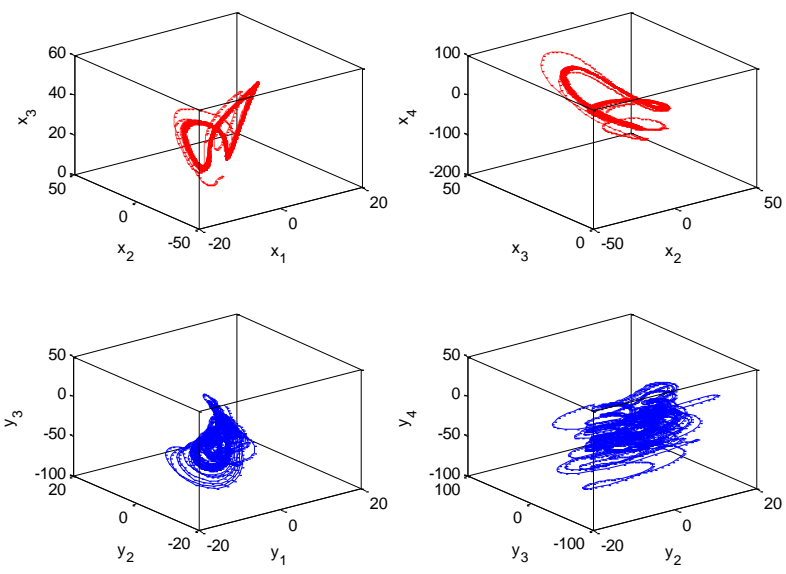

Figure 1. Chaotic Attractors of Drive System (35) and Response System (36)

For observing the control effect of the proposed method, we firstly given the state trajectories of the drive system and response system without controller, shown in Figure 2.
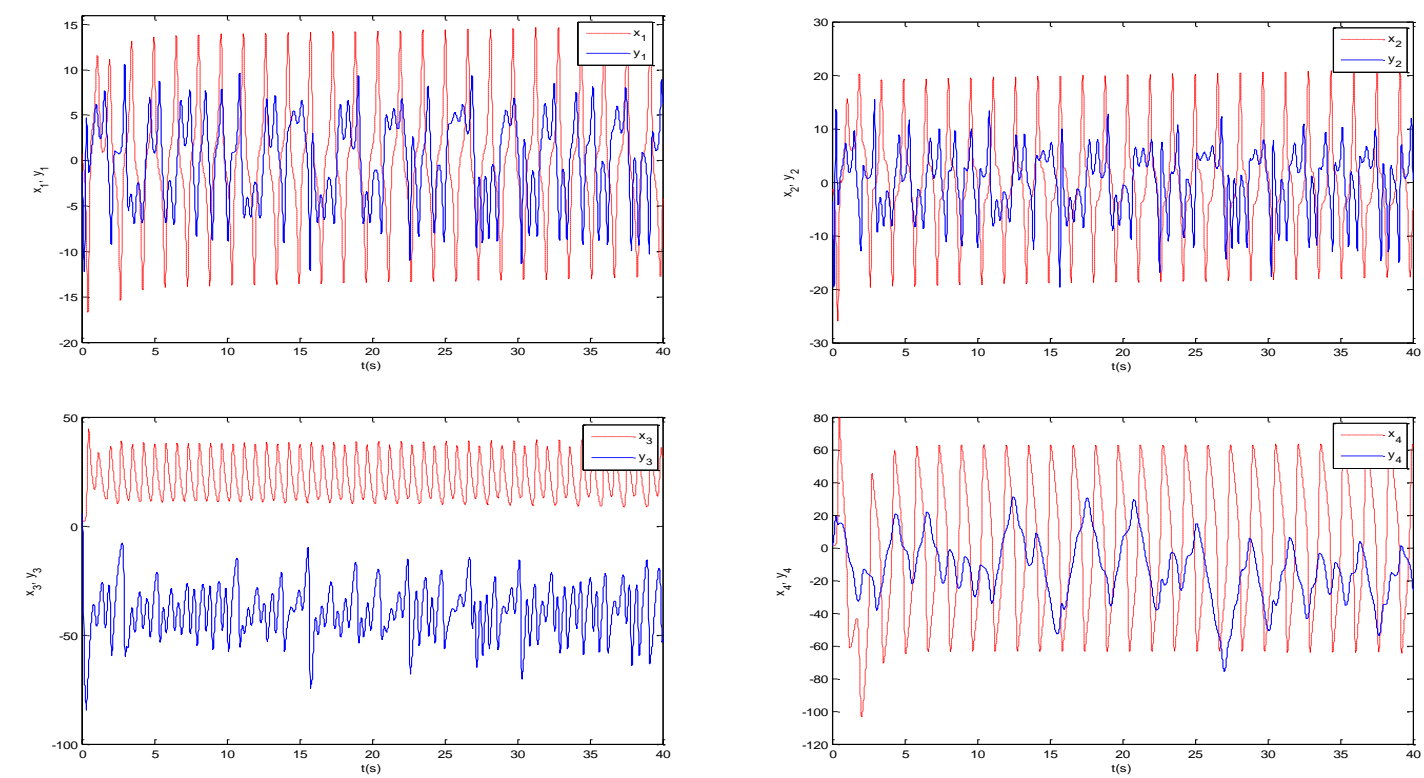

Figure 2. State Trajectories of Drive and Response Systems without Controller

From systems (35) and (36), we know that

$$
A_{1}=\left(\begin{array}{cccc}
-a & a & 0 & 1 \\
c & -1 & 0 & 0 \\
0 & 0 & -b & 0 \\
0 & 0 & 0 & -r
\end{array}\right), f_{1}(x)=\left(\begin{array}{c}
0 \\
-x_{1} x_{3} \\
x_{1} x_{2} \\
-x_{2} x_{3}
\end{array}\right)
$$




$$
A_{2}=\left(\begin{array}{cccc}
-a_{1} & a_{1} & 0 & 0 \\
q & 0 & 0 & c_{1} \\
0 & 0 & -b_{1} & -d_{1} \\
-r_{1} & 0 & 0 & 0
\end{array}\right), f_{2}(y)=\left(\begin{array}{c}
0 \\
y_{1} y_{3} \\
-2 y_{1}^{2}-2 y_{2}^{2} \\
0
\end{array}\right)
$$

According to (14), for convenience, we can choose $B=(1,1,0,0)^{T}$, the synchronization error dynamics is described as

$$
\begin{aligned}
& D^{\beta_{1}} e_{1}=a_{1}\left(e_{2}-e_{1}\right)+w(t) \\
& D^{\beta_{2}} e_{2}=q e_{1}+c_{1} e_{4}+w(t) \\
& D^{\beta_{3}} e_{3}=-b_{1} e_{3}-d_{1} e_{4} \\
& D^{\beta_{4}} e_{3}=-r_{1} e_{1}
\end{aligned}
$$

From Theorem 1, $C$ can be selected as $C=(20,0,1,0)$, then the eigenvalues of $A_{e q}=\left(I-B(C B)^{-1} C\right) A_{2} \quad$ are $\quad \lambda_{1}=-10 \quad, \quad \lambda_{2}=-7.216 \times e^{-16}, \quad \lambda_{3}=-0.2192$, $\lambda_{4}=-2.2808$, which satisfy the condition $\left|\arg \left(\operatorname{spec}\left(A_{e q}\right)\right)\right|>\alpha \pi / 2$, so the value of $\left|\arg \left(\operatorname{spec}\left(A_{e q}\right)\right)\right|$ lies in the stable region. By Theorem 1 , choose parameters $k_{1}=1$, $k_{2}=1, k_{3}=1, k_{4}=4$. With the sampling time $h=0.01 s$, then we can get the time response of synchronization error system (37) with the controller activated at $t=10 \mathrm{~s}$, illustrated in Figure 3.
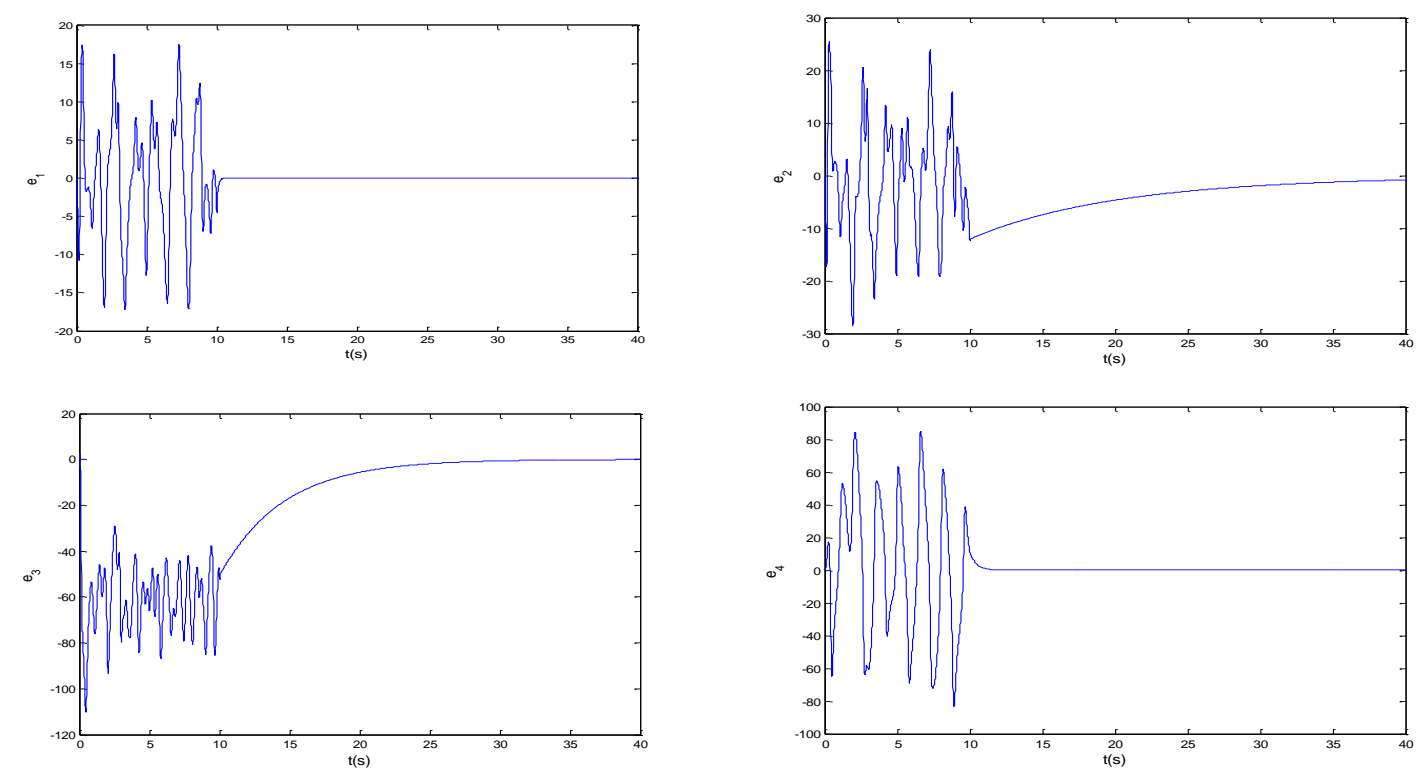

Figure 3. Time Response of the Error System with Controller Activated at $t=10 \mathrm{~s}$

In Figure 3, it is not hard to see, the synchronization errors converge to zero asymptotically, which implies that the SOSM approach is effective to address the synchronization problem of two different fractional order hyperchaotic systems. The state trajectories of drive and response systems with the controller activated at $t=10 \mathrm{~s}$ are displayed in Figure 4. 

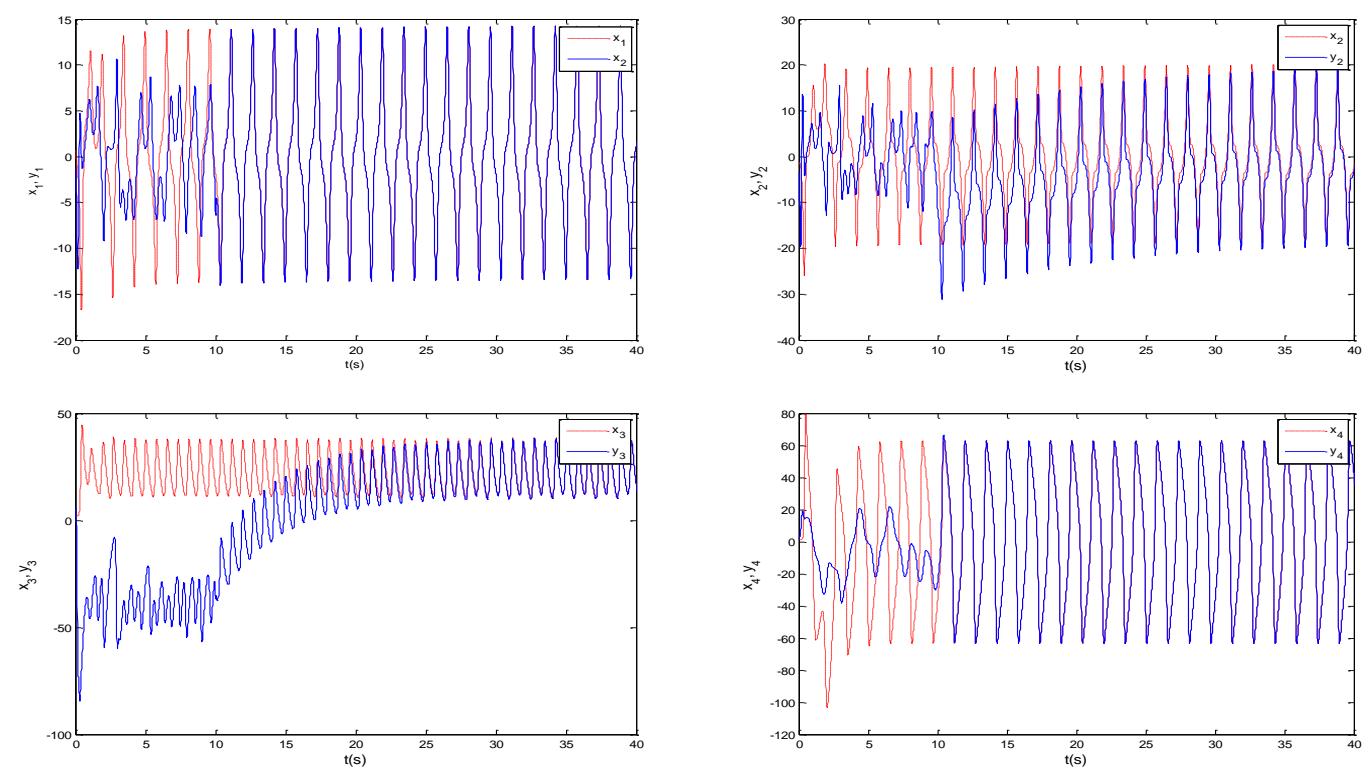

Figure 4. Time Evolutions of Drive System and Response System with Controller Activated at $t=10 \mathrm{~s}$

From Figure 4, it is easily can be seen that with applying the proposed robust controller, the state trajectories of the response system (36) asymptotically converge to the drive system (35).

These simulation results verify the good robustness of the proposed approach and highlight the better performance featured by SOSM technique.

\section{Conclusions}

The modified SOSM scheme is proposed in this paper to synchronize two different fractional order hyperchaotic systems. A special fractional order sliding mode manifold is firstly designed, and the design procedures are described in detail. Subsequently, on the basis of modified SOSM technique and finite-time theory, a controller is designed to ensure the occurrence of sliding motion in finite time. Finally, the stability of the suggested scheme has been demonstrated via Lyapunov theorem, and a simulation example is given to show its effectiveness.

\section{Acknowledgements}

The author would like to thank the reviewers for their careful reading and providing some pertinent suggestions. This work is supported by the National Natural Science Foundation of China $(61273119,61374038)$ and the Nature Science Foundation of Jiangsu Province of China (BK2011253).

\section{References}

[1] R. L. Bagley and R. A. Calico, J. Guid. Control Dynam., vol. 14, (1991).

[2] H. H. Sun, A. A. Abdelwahad and B. Oharal, "IEEE T. Autom. Contr.", vol. 29, (1984).

[3] M. Ichise, Y. Nagayanagi and T. Kojima, "J. Electroanal. Chem. Interfacial Electrochem.", vol. 33, (1971).

[4] O. Heaviside, Electromagnetc Theory, Cambridge University Press, Cambridge (1971).

[5] R. X. Zhang and S. P. Yang, "Nonlinear Dynam.", vol. 68, (2012).

[6] J. G. Lu, "Physica A", vol. 359, (2006).

[7] H. Targhvafard and G. H. Erjaee, "Commun. Nonlinear Sci. Numer. Simul.", vol. 16, (2011).

[8] Z. M. Odibat, "Nonlinear Dynam.”, vol. 60, (2010). 
[9] D. Y. Chen, R. F. Zhang, and J. C. Sprott, "Chaos", vol. 22, (2012).

[10] D. Y. Chen, R. F. Zhang, and X. Y. Ma, “Chin. Phys. B.”, vol. 21, (2012).

[11] M. P. Aghababa, S. Khanmohammadi, and G. Alizadeh, "Appl. Math. Model.”, vol. 35, (2011).

[12] S. P. Bhat and D. S. Bernstein, "SIAM J.", Control Optim., vol. 38, (2000).

[13] A. Pisano, M. R. Rapaic, Z. D. Jelicic, and E. Usai, “Int. J. Robust Nonlin. Contr.”, vol. 20, (2010).

[14] L. P. Chen, Y. Chai, R. C. Wu, J. Sun, and T. D. Ma, "Phys. Lett. A", vol. 376, (2012).

[15] M. S. Tavazoei and M. Haeri, "Phys. Lett. A", vol. 387, (2008).

[16] K. Diethelm and N. Ford, "Nonlinear Dynam.", vol. 29, (2002).

[17] N. N. Yang and C. X. Liu, "Nonlinear Dynam.", vol. 74, (2013).

[18] H. Chen, W. Chen, B. W. Zhang, and H. T. Cao, "J. Appl. Math.”, (2013). 